\title{
Cold Ischemia Procedure
}

National Cancer Institute

\section{Source}

National Cancer Institute. Cold Ischemia Procedure. NCI Thesaurus. Code C116556.

The act of decreasing both the temperature and blood flow to an organ or tissue for the purpose of preserving it for transplantation. 